\title{
The Neutral Transport Analysis Based on Visible Light Measurement of Recycling and 3-Dimensional Simulation in GAMMA 10
}

\author{
Yuta HIGASHIZONO, Yousuke NAKASHIMA, Mamoru SHOJI ${ }^{1)}$, Shinji KOBAYASHI ${ }^{2}$, \\ Nobuhiro NISHINO ${ }^{3}$, Hirokazu KAWANO, Yuusuke KUBOTA, Takayuki KOBAYASHI, \\ Masayuki YOSHIKAWA, Yoshiyuki MISHIMA, Mirai SUZUKI, Keiji MORI and Teruji CHO \\ Plasma Research Center, University of Tsukuba, Tsukuba, Ibaraki 305-8577, Japan \\ 1) National Institute for Fusion Science, Toki, Gifu 509-5292, Japan \\ 2) Institute of Advanced Energy, Kyoto University, Gokasho, Uji 611-0011, Japan \\ ${ }^{3)}$ Graduate School of Engineering, Hiroshima University, Hiroshima 739-8527, Japan
}

(Received 5 December 2006 / Accepted 2 April 2007)

\begin{abstract}
Neutral transport related to recycling phenomena on two limiters installed in the central-cell was studied in the GAMMA 10 tandem mirror plasmas. Dependence of iris limiter is investigated in terms of plasma diamagnetism and $\mathrm{H}_{\alpha}$-line intensity. It was found that a plasma diamagnetic signal was improved and $\mathrm{H}_{\alpha}$-line intensity near the iris limiter increased if the diameter of the iris limiter shrank to $340 \mathrm{~mm}$. Fully 3-dimensional MonteCarlo simulation (DEGAS) was executed with the recycling source on the limiters. The neutral sources on the limiters were consistently given so as to satisfy 2-dimensional (2-d) image obtained by a high-speed camera. The determined $\mathrm{H}_{\alpha}$-line intensity from DEGAS well explained the experimental result. The simulation result, in the $340 \mathrm{~mm}$ case, predicted the reduction in the peak value of $\mathrm{H}_{\alpha}$-line intensity on the central limiter and the enhancement on the iris limiter. It suggests that the reduction of the recycling on the central limiter makes it possible to lead the better plasma performance.
\end{abstract}

(C) 2007 The Japan Society of Plasma Science and Nuclear Fusion Research

Keywords: GAMMA 10, tandem mirror, neutral transport, Monte-Carlo simulation, DEGAS, limiter, hydrogen recycling, $\mathrm{H}_{\alpha}$-line intensity, 2-d image

DOI: $10.1585 /$ pfr.2.S1087

\section{Introduction}

In magnetic confining fusion devices, particle balances are important issues for obtaining good plasma performances. Neutral particles induced by limiters, divertors, gas puffers and neutral beam injection (NBI) must be controlled on an appropriate quantity. Recycling phenomena on limiters have a more significant influence on plasma performance compared with those on the other vessel wall, since the limiters are located near the plasma boundary. The detailed analysis of neutral transport by the passive source such as limiters enables us to control the active neutral source such as gas puffers [1,2].

In the GAMMA 10 central-cell, central limiter and iris limiter have been installed, the former is a fixed type of limiter and the latter is a radially variable type of limiter, respectively. Electron cyclotron resonance heating systems (ECRH) are installed on the central and plug/barrier cells. These are used for heating bulk electrons and producing plasma confinement potential [3].

In order to measure neutral particles, $\mathrm{H}_{\alpha}$-line detectors and high-speed camera have been installed $[4,5]$. Axially and radially aligned $\mathrm{H}_{\alpha}$-line detectors take advantage of the spatial distribution. The high-speed camera has been also mounted on the central mid-plane. It is sufficiently capable of measuring the light emission with a band of wavelength in visible ranges. Based on these measurements, the detailed neutral distribution is predicted by utilizing Monte-Carlo simulation code (DEGAS) [6, 7]. In the mesh-model for DEGAS, not only the vacuum vessel but also the inner structures including the central limiter and the iris limiter have been completely realized. The $\mathrm{H}_{\alpha}$ emission on the each mesh is numerically solved using atomic and molecular hydrogen density.

Recently, the diameter of the iris limiter has been properly changed fitting the experimental objective, which has controlled the plasma condition. Especially, drastic improvements of the plasma performance were observed during ECRH in the central-cell (c-ECRH) by optimizing the diameter of the iris limiter. In this paper, axial $\mathrm{H}_{\alpha}$-line intensity and the plasma parameters are analyzed in terms of the iris limiter. Moreover, using DEGAS mesh-model, the $\mathrm{H}_{\alpha}$-line intensity distribution due to the recycling on the central limiter and the iris limiter is quantitatively estimated. Finally, appropriate recycling supplies for plasma by the neutral source are discussed. 


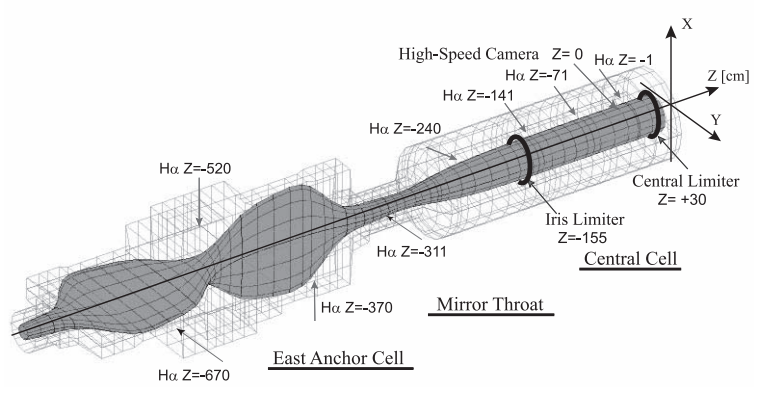

Fig. 1 The schematic view of Ha-line detectors and high-speed camera. The plasma and vacuum vessel structure and the limiters are illustrated.

\section{Experimental Setup}

The GAMMA10 tandem mirror is an open magnetic plasma-confining device with thermal barrier [8]. It consists of central-cell, anchor-cells, plug/barrier-cells and end-cells. Mid-plane of the central-cell is $Z=0 \mathrm{~cm}$ and west and east side corresponds to plus and minus in $\mathrm{Z}$ axis, respectively. Central-cell is the main region to confine plasma and is $6 \mathrm{~m}$ in length and the diameter of $1 \mathrm{~m}$. Mirror-throat regions which exist on between the centralcell and each anchor-cell have the first mirror with strong magnetic field for the confining plasma in the central-cell. In standard hot-ion mode plasmas, initial plasma is built up by plasma guns located in both ends. Then plasma is sustained by gas puffing coupling with ion cyclotron heating. c-ECRH and neutral beam injection (NBI) [9] are additionally supplied for the plasma production and heating. A typical electron density reaches $4 \times 10^{12} \mathrm{~cm}^{-3}$ in the centralcell.

Figure 1 shows the schematic view of the axially aligned $\mathrm{H}_{\alpha}$-line detectors, the high-speed camera and the location of the central limiter $(Z=+30 \mathrm{~cm})$ and the iris limiter $(Z=-155 \mathrm{~cm})$ together with magnetic flux tube and the vacuum vessel. In the central-cell, magnetic flux tube is expanded in the mid-plane, and shrinks toward the both mirror-throat regions. The central limiter is the fixed type of limiter and $360 \mathrm{~mm}$ in diameter, while the iris limiter is radially variable type of limiter. Each axially aligned $\mathrm{H}_{\alpha}$-line detector in the central-cell has been located at $Z=-1,-71,-141,-240$ and $-311 \mathrm{~cm}$, respectively. A microwave for c-ECRH is launched in the mirror-throat region on the east side and the microwave power is absorbed near a fundamental harmonic region around $Z=-240 \mathrm{~cm}$.

\section{Experimental Results}

Figure 2 shows the temporal distribution of plasma parameters and $\mathrm{H}_{\alpha} / \mathrm{NLcc}$ which means the $\mathrm{H}_{\alpha}$-line intensities divided into electron line density measured in the centralcell (NLcc) in the cases that the diameters of the iris limiter are $400 \mathrm{~mm}$ and $340 \mathrm{~mm}$. It is obvious that diamagnetic signal (DMcc) and NLcc immediately drop after c-ECRH
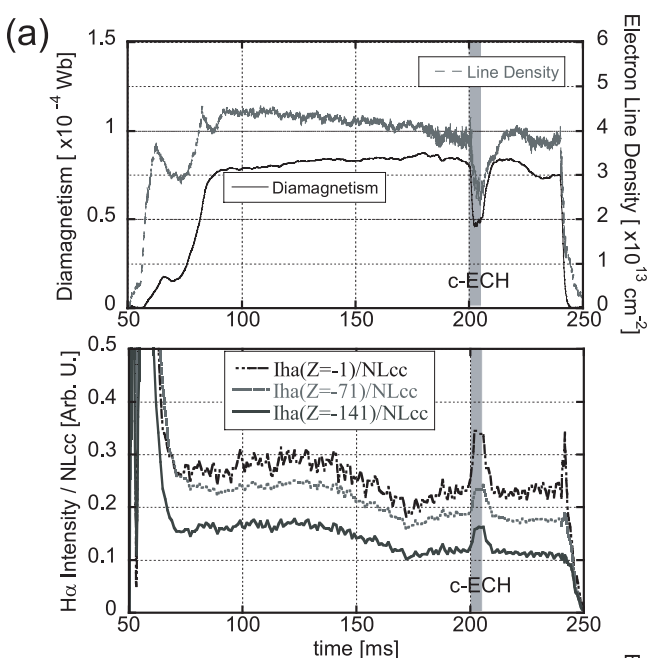

(b)
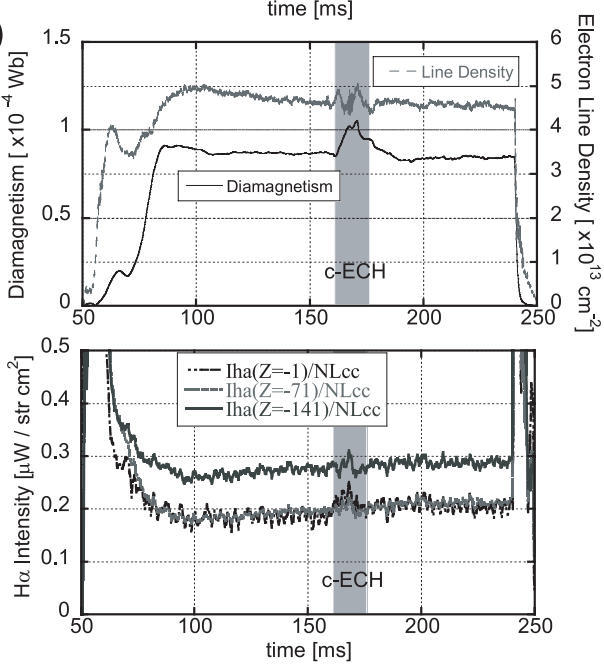

Fig. 2 The temporal distributions of plasma parameters and Haline intensity in the central-cell in the case of $400 \mathrm{~mm}$ (a) and $340 \mathrm{~mm}$ (b) in diameter of the iris limiter.

in the case of $400 \mathrm{~mm}$. At the same time, $\mathrm{H}_{\alpha} / \mathrm{NLcc}$ remarkably increase, especially in $Z=-1 \mathrm{~cm}$ where locate near the central limiter. In the whole plasma duration, $\mathrm{H}_{\alpha} / \mathrm{NLcc}$ in $Z=-1 \mathrm{~cm}$ remains at the highest level, followed by $Z=-71 \mathrm{~cm}$ and $Z=-141 \mathrm{~cm}$. In case that the diameter of iris limiter became $340 \mathrm{~mm}$, on the other hand, DMcc gradually increases together with c-ECRH and then the $\mathrm{H}_{\alpha}$-line intensities hardly change during c-ECRH. It is found that $\mathrm{H}_{\alpha} / \mathrm{NLcc}$ in $Z=-141 \mathrm{~cm}$ is kept in higher value than that in $Z=-1 \mathrm{~cm}$.

The relationship between the $\mathrm{H}_{\alpha}$-line intensity in $Z=$ $-1 \mathrm{~cm}$ and that in $Z=-141 \mathrm{~cm}$ was examined in regard to the diameter of the iris limiter. In Fig. 3, the rate of the $\mathrm{H}_{\alpha^{-}}$ line intensity in $Z=-141 \mathrm{~cm}$ to $Z=-1 \mathrm{~cm}$ is plotted as a function of the diameter of the iris limiter. It is confirmed that this rate intends to increase according to the reduction of the diameter of the iris limiter.

\section{Simulation Method}

Monte Carlo simulation code (DEGAS) has been 


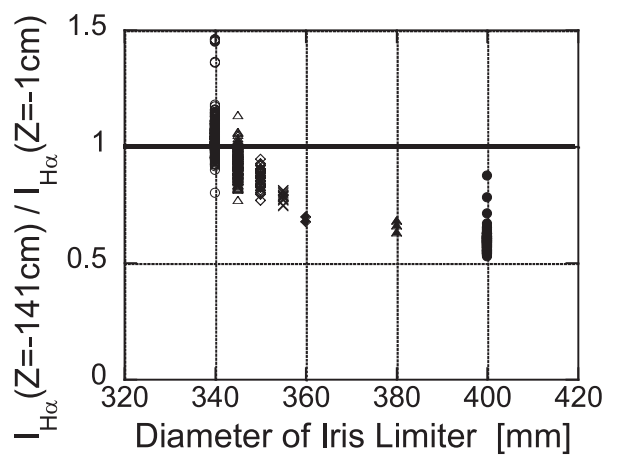

Fig. 3 The rate of Ha-line intensity in $Z=-141 \mathrm{~cm}$ and $Z=$ $-1 \mathrm{~cm}$ versus the diameter of the iris limiter.

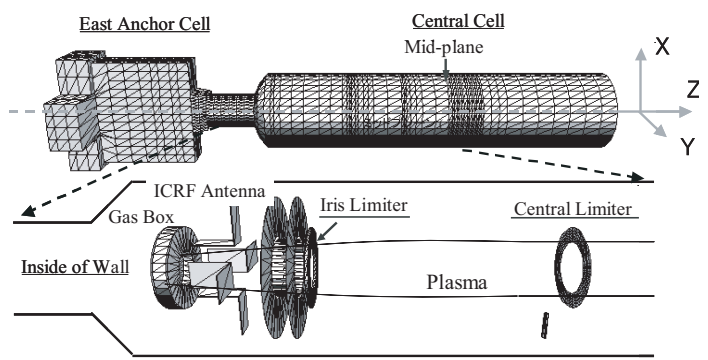

Fig. 4 The fully 3-dimensional DEGAS mesh-model.

used in order to numerically calculate the neutral density coupling with the measurement of $\mathrm{H}_{\alpha}$-line detectors and photographs by high-speed camera. Fully 3-dimensional mesh-model for DEGAS has been applied shown in Fig. 4. In this model of the central-cell, the limiters, antennas of ion-cyclotron radio frequency (ICRF) and gas puffers are precisely implemented in realistic configuration. Test particles defined as neutral hydrogen is randomly passing through the plasma or SOL in consideration of physical process. After that, atomic and molecular hydrogen densities are resolved at the each mesh. For the granular simulation of the recycling on the limiters, the iris limiter was newly added and the mesh of the central limiter separated in the large number.

\section{Comparison Between Experiment and Simulation}

Based on the 2-d images captured near the central limiter by using the high-speed camera, it was determined how to give test particles of the Monte-Carlo simulation on the limiters. The test particles defined as neutral hydrogen is initially given on the mesh of limiters to satisfy the 2-d image obtained by high-speed camera. As shown in Fig. 5 (a) and (b), the 2-d image of $\mathrm{H}_{\alpha}$-line intensity calculated by DEGAS is consistent with that by the high-speed camera.

The simulation was carried out in two cases that the diameter of the iris limiter is $400 \mathrm{~mm}$ and $340 \mathrm{~mm}$. The neutral source was given in the central limiter and the iris limiter. Figure 6 shows the axial distribution of the $\mathrm{H}_{\alpha^{-}}$ (a)

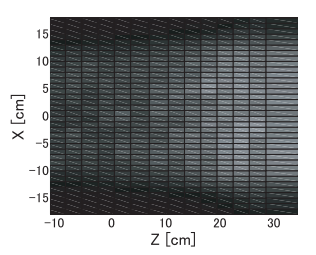

(b)

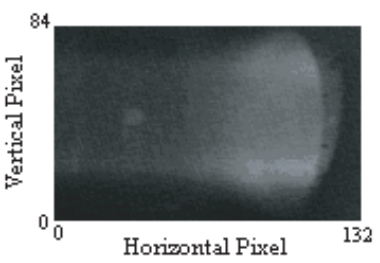

Fig. 5 2-d images by Ha calculation in the DEGAS simulation (a) and by high-speed camera (b) around the central limiter.

(a)

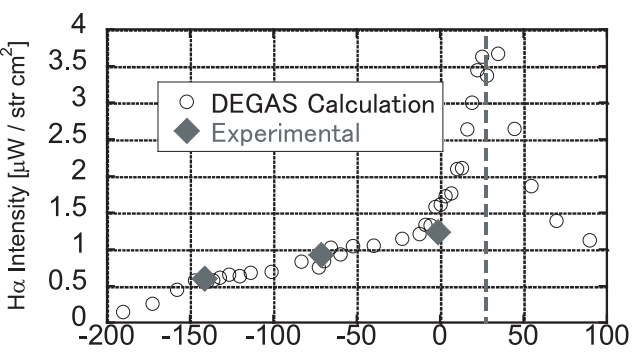

(b)

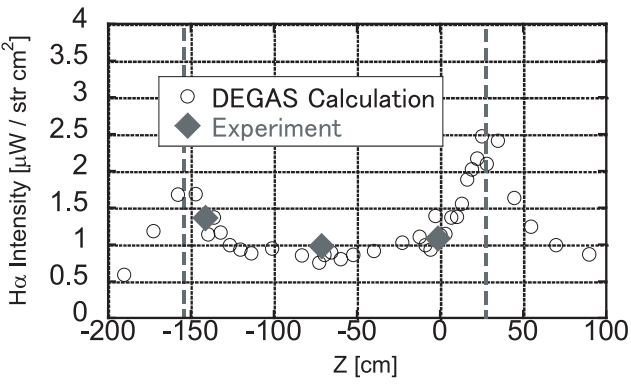

Fig. 6 The Ha-line intensity of the DEGAS simulation and the experiment in the case of $400 \mathrm{~mm}$ (a) and $340 \mathrm{~mm}$ (b) in diameter of the iris limiter.

line intensity obtained by the experiment and predicted from the DEGAS simulation. In cases that the diameter of iris limiter is $400 \mathrm{~mm}$ (Fig. 6(a)), the simulation results are appropriately corresponding with the measurement if the neutral source is given only from the central limiter in the simulation. On the other hand, in the case of $340 \mathrm{~mm}$ (Fig. 6(b)), both the central limiter and the iris limiter are defined as neutral source, which leads to the proper $\mathrm{H}_{\alpha^{-}}$ line intensity in axial distribution. Therefore, the $\mathrm{H}_{\alpha}$-line intensity profiles by DEGAS have two peaks on the central limiter and the iris limiter. Each peak shows a lower value than the one peak on the central limiter in the case of $400 \mathrm{~mm}$.

\section{Discussion}

From the experimental and simulation results, it suggests that the iris limiter and the central limiter have much influence on the plasma condition and the neutral transport. It is certain that the iris limiter become a large number of neutral sources, as the diameter of the iris limiter is getting smaller. As the result, another peak of $\mathrm{H}_{\alpha}$-line intensity 
(a)

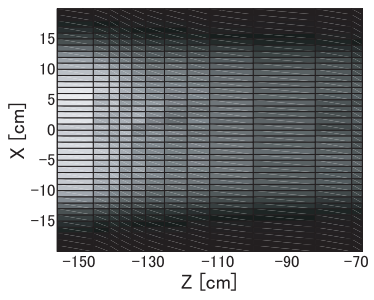

(b)

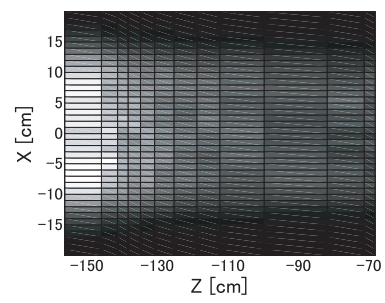

Fig. 7 2-d images by DEGAS around the iris limiter in the cases of $380 \mathrm{~mm}$ (a) and $340 \mathrm{~mm}$ (b).

distribution is formed around the iris limiter except around the central limiter. And then, it notes that the measurements of $\mathrm{H}_{\alpha} / \mathrm{NLcc}$ in $Z=-1 \mathrm{~cm}$ are reduced and the peak around the central limiter of the simulation obviously decreases, if the iris limiter shrink from $400 \mathrm{~mm}$ to $340 \mathrm{~mm}$ in diameter.

The plasma in the central mid-plane has been sustained with high density and high energy compared to other positions. From the analysis of the high speed camera, rotations and vibrations of the plasma column during cECRH have been confirmed. Therefore, the excessive recycling source on the central limiter, especially during cECRH, maybe causes the degradation of the plasma performance. Consequently, it must be important for the plasma performance to decrease the recycling source on the central limiter such as in the case of $340 \mathrm{~mm}$.

In order to estimate the effect of the iris limiter on the neutral transport, the localization of the $\mathrm{H}_{\alpha}$-line intensity related to the recycling source on the iris limiter was examined. This simulation was executed in the case of $380 \mathrm{~mm}$ and $340 \mathrm{~mm}$ in diameter of the iris limiter. Figure 7 shows the 2-d images of the calculated $\mathrm{H}_{\alpha}$-line intensity around the iris limiter in the both cases. The image in the $340 \mathrm{~mm}$ case is more enhanced at the near position of the iris limiter and reduced at distant locations compared with the $380 \mathrm{~mm}$ case. It is clarified that $\mathrm{H}_{\alpha}$-line intensity is much localized near the iris limiter if the diameter becomes narrower.

From the above results, it is expected that the local- ization of the $\mathrm{H}_{\alpha}$-line intensity distribution on the limiters should be concerned with neutral transport. In future, in order to estimate the more detailed behavior of neutral particles around the limiters, the visible light measurements will be required in the nearer position of limiters.

\section{Summary}

Neutral transport related to the recycling phenomena on the limiters in the central-cell was analyzed. From the experiment, it was observed that the plasma diamagnetic signal increased in cases that the diameter of the iris limiter is $340 \mathrm{~mm}$. The rate between $\mathrm{H}_{\alpha}$-line intensity in $Z=-141$ $\mathrm{cm}$ and that in $Z=-1 \mathrm{~cm}$ proved to increase along with the reduction of the diameter size of the iris limiter.

The Monte-Carlo simulation was executed for the calculation of neutral transport due to recycling source on the limiters. In the case of $340 \mathrm{~mm}$, the $\mathrm{H}_{\alpha}$-line intensity distribution by DEGAS has two peaks on the central and the iris limiter. Each peak takes a lower value than that of the central limiter on the condition of $400 \mathrm{~mm}$. It suggests that the reduction of the recycling on the central limiter results in the improvement of the plasma performances.

\section{Acknowledgement}

This study is partly supported by the bi-directional collaboration researches with NIFS (NIFS04KUGM008). The authors would like to thank the members of the GAMMA10 groups for their collaboration in the experiments and for helpful discussion.

[1] T. Nakashima et al., J. Nucl. Mater. 196-198, 493 (1992).

[2] S. Kobayashi et al., J. Nucl. Mater. 266-269, 566 (1999).

[3] T. Cho et al., Nucl. Fusion 45, 1650 (2005).

[4] Y. Higashizono et al., Trans. Fusion Technol. 47 No.1T, 297 (2005).

[5] N. Nishino et al., Plasma Fusion. Res. 1, 035 (2006).

[6] D.B. Heifetz et al., J. Comp. Phys. 46, 309 (1982).

[7] D.P. Stotler et al., Phys. Plasmas. 3, 4084 (1996).

[8] M. Inutake et al., Phys. Rev. Lett. 55, 939 (1985).

[9] Y. Nakashima et al., J. Nucl. Mater. 313-316, 553 (2003). 